\title{
KINEMATIC ANALYSIS OF JAW MOVEMENTS
}

\author{
CIORTAN, M[arinela]
}

\begin{abstract}
The jaw can be moved directly forward or backward; up and down, a pure hinge motion; a rotary movement on a vertical axis through one of the condyles; and rotation on a transverse axis passing from side to side through the mandibular or inferior dental foramina. To simulate the movement of the mandible can be achieved using a kinematics analysis of upper joints. The kinematics analysis of the mechanism is to determine the parameters of position, velocity and acceleration corresponding to all elements.

Key words: mandible, muscle, movements, kinematics
\end{abstract}

\section{INTRODUCTION}

The mandible has four basic movements. The jaw can move directly forward or backward; up and down, a pure hinge motion; a rotary movement on a vertical axis through one of the condyles; and rotation on a transversal axis passing from side to side through the mandibular or inferior dental foramina.

Movement can be decomposed and studied in the three reference planes: frontal, sagittal and horizontal.

The mandible movements you make can be classified into: basic movements, movements composed of fundamental movements and functional movements.

The incremental movements are the translational and rotational movements, which can run in joint cavities, synchronous and asynchronous. The rotation movement is performed on the main axis of rotation of the temporomandibular joint, and rotation can be run around a vertical axis or sagittal - the lateral excursion. The operation involvs more jaw muscle groups.

Thus, the lifting movement of the jaw (mouth closed), the masseter muscle has the essential role, also the temporal muscle and the pterygoid muscle help. Braking is done with muscle movement: posterior digastric, digastric and sternohyoid previously.

\section{DESCRIPTION OF METHOD}

Rotation around the temporomandibular jaw joint, called the medical literature and hinge motion is carried around an imaginary axis called bicondilian shaft, passing through two of the mandibular condyle. It is estimated that the rotation is ideally produces an opening angle of $12^{\circ}$ and possesses all the properties of speed and acceleration distribution for fixed axis rotation. In this batch?, angular velocity $\omega$ is expected to be constant. Rotation movement of the mandible occurs naturally and is easy to put out.

\subsection{Upper kinematics couplings}

Simulation of the movement of the mandible can be achieved using a kinematics analysis of upper joints. Figure 1 shows the two profiles (of the mandible and skull) in contact at point $C$. The role of the kinematics analysis of the mechanism is to determine the parameters of position, velocity and acceleration corresponding to all elements. To determine these parameters, calculation procedures are used, prepared for each module. For this purpose, it is necessary to determine the components of the mechanism module which simulate the jaw movement.

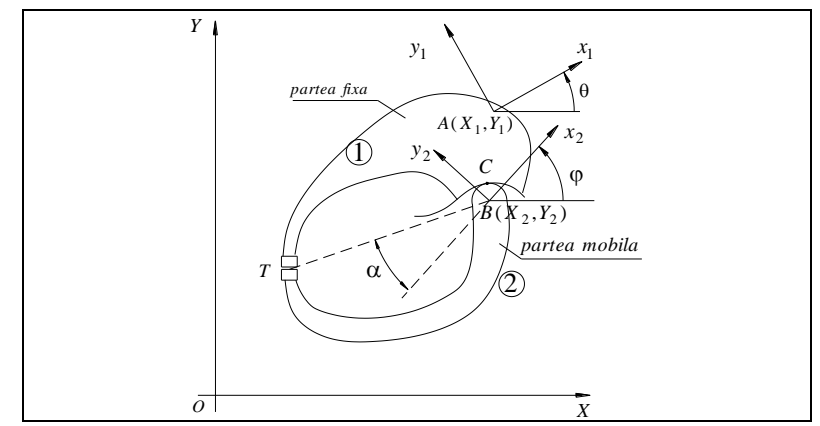

Fig. 1. Representation of the masticator apparatus using kinematics couplings

To obtain analytical expressions of the equations of a parametric profile 1 measurement on the skull to determine the numerical values of these coordinates, then by the method of least squares, determine the analytical expression of the function that best approximates these coordinates.

The laws of motion of the two profiles are given by roots movements and systems of axes $A x_{1} y_{1}$ and $B x_{2} y_{2}$, and rotations of these systems to XOY fixed axis system, namely:

-the law of motion of a profile

$$
\begin{aligned}
& X_{1}=X A=\text { const }, \\
& Y_{1}=Y A=\text { const } \\
& \theta=\text { const },
\end{aligned}
$$

-the law of motion of profile 2 (the law of motion of the mandible).

$$
\left\{\begin{array}{l}
X_{2}=X B(t) ; \\
Y_{2}=Y B(t) ; \\
\varphi=\varphi(t) ;
\end{array}\right.
$$

Parametric equations of (arcs)curves of the two sections of fulltime guidelines are:

-the parametric equations of a profile 1 (you chose a 5degree polynomial, you can choose and other equations?)

$$
\left\{\begin{array}{l}
x_{1}=x_{1} ; \\
y_{1}=a_{0}+a_{1} x_{1}+a_{2} x_{1}^{2}+a_{3} x_{1}^{3}+a_{4} x_{1}^{4}+a_{5} x_{1}^{5} ;
\end{array}\right.
$$

-the parametric equations of profile 2 are:

$$
\left\{\begin{array}{l}
x_{2}=R \cos (v) ; \\
y_{2}=R \sin (v) ;
\end{array}\right.
$$

where: $v$ is the parameter generating? crupper (it was considered an arc centred on point B) 
The condition of contact between the two sections of fulltime 1 and 2, XOY fixed system is given by:

$$
\begin{aligned}
& X_{10}=X_{20}=X_{C} ; \\
& Y_{10}=Y_{20}=Y_{C},
\end{aligned}
$$

where:

$$
\begin{aligned}
& X_{10}\left(x_{1}, t\right)=X A+x_{1} \cos \theta-y_{1} \sin \theta \\
& Y_{10}\left(x_{1}, t\right)=Y A+x_{1} \sin \theta+y_{1} \cos \theta,
\end{aligned}
$$

the transformation of the coordinates of a point fixed system $X O Y$ of the mobile system $A x_{1} y_{1}$ and

$$
\begin{aligned}
& X_{20}(v, t)=X B+R \cos (v) \cos (\varphi)-R \sin (v) \sin (\varphi) ; \\
& Y_{20}(v, t)=X B+R \sin (v) \sin (\varphi)+R \cos (v) \cos (\varphi) ;
\end{aligned}
$$

the transformation of the coordinates of a point fixed system $X O Y$ of mobile system $B x_{2} y_{2}$.

The tangency condition in the same fixed system XOY is:

$$
\frac{\frac{\partial Y_{10}}{\partial x_{1}}}{\frac{\partial X_{10}}{\partial x_{1}}}=\frac{\frac{\partial Y_{20}}{\partial v}}{\frac{\partial X_{20}}{\partial v}},
$$

where:

$$
\begin{gathered}
\frac{\partial X_{10}}{\partial x_{1}}=\cos (\theta)-\frac{d y_{1}}{d x_{1}} \sin (\theta) ; \\
\frac{\partial Y_{10}}{\partial x_{1}}=\sin \theta+\frac{d y_{1}}{d x 1} \cos (\theta) ; \\
\frac{\partial X_{20}}{\partial v}=-R \sin (v) \cos (\varphi)-r \cos (v) \sin (\varphi) ; \\
\frac{\partial Y_{20}}{\partial v}=-R \sin (v) \sin (\varphi)+\frac{d y_{2}}{d v} \cos (\varphi) .
\end{gathered}
$$

The parameter $v$ is introduced in the relation equations (7) and the resulting coordinates of point $B$

$$
\begin{aligned}
& X_{20}=X B+R \cos \left(\theta-\varphi+\arctan \left(\frac{-1}{\frac{d y}{d x_{1}}}\right)+\pi\right) \cdot \cos (\varphi)- \\
& -R \sin \left(\theta-\varphi+\arctan \left(\frac{-1}{\frac{d y}{d x_{1}}}\right)+\pi\right) \cdot \sin (\varphi) ; \\
& Y_{20}=X B+R \sin \left(\theta-\varphi+\arctan \left(\frac{\frac{-1}{d y}}{d x_{1}}\right)+\pi\right) \cdot \sin (\varphi)+ \\
& +R \cos \left(\theta-\varphi+\arctan \left(\frac{-1}{\frac{d y}{d x_{1}}}\right)+\pi\right) \cdot \cos (\varphi),
\end{aligned}
$$

which is the origin of the mobile system $B x_{2} y_{2}$ in the coordinate system $X O Y$. By modifying the values of the angle $\varphi$, the position of the reinsurance determine the coordinates of point $\mathrm{B}$ and the parameter $\varphi$ can be determined using nonlinear system $\mathrm{T}$ of equations consisting of equations (5) and (8).

\subsection{Lower kinematics couplings}

Kinematics analysis of jaw and can be done using equivalent mechanisms such as in Figure 2.

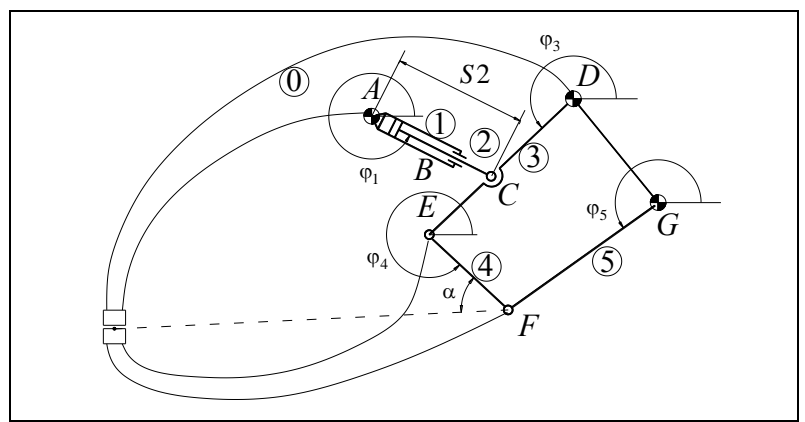

Fig. 2. Lower coupling mechanism

Taking into account the relative movements of the elements, it appears that the lower coupling mechanism is the following:

$A(1 R 0), B(1 T 2), C(2 R 3), D(3 R 0), E(3 R 4), F(4 \mathrm{R} 5), G(5 R 0)$

Higher number is zero couplings.

Mobile elements of the mechanism are:

Given the number of moving parts kinematics couplings, resulting degree of mobility of the mechanism, namely: $\mathrm{M}=1$.

Structural schemes and multi-polar mechanism in figure 3 are shown in figures 3.a and 3.b. Figure 3.c is presenting the structural relationship of the mechanism.

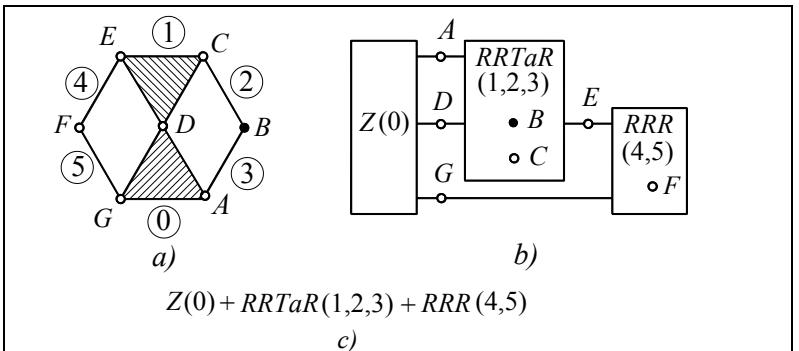

Fig. 3. Structural scheme of lower kinematics coupling mechanism

\section{CONCLUSION}

Jaw movement can be simulated [using different types of kinematics couplings.

\section{REFERENCES}

Bratu D. şi colectivul - Aparatul dento-maxilar. Date de morfologie funcţională clinică, Editura Helicon Timişoara, 1997

Doina Dragulescu - Grant - Studierea dinamicii mandibulei şi modelarea de implante pentru corectarea prin intervenţii chirurgicale a ruperii sale accidentale - Universitatea Politehnică Timişoara

Dreucean Mircea Conf. dr. ing. - Modelarea, proiectarea si realizarea practica a unui sistem de implanturi medicale destinat chirurgiei maxilo-faciale si ortopedice Universitatea: Universitatea POLITEHNICA din Timisoara

Papillian V.: Anatomia omului, vol. 1-2, ediţia a V-a, E.D.P. Bucureşti, 1974 